Homology, Homotopy and Applications, vol. 7(3), 2005, pp.37-52

\title{
ENTIRE CYCLIC COHOMOLOGY OF SCHATTEN IDEALS
}

\section{JACEK BRODZKI AND ROGER PLYMEN}

(communicated by J.F. Jardine)

\author{
Abstract \\ We compute the entire cyclic homology and cohomology of \\ the Schatten ideals $\mathfrak{L}^{p}$. \\ Dedicated to Victor Snaith on the occasion of his 60-th birthday.
}

\section{Introduction}

This paper is devoted to the computation of the entire cyclic cohomology of Schatten ideals $\mathfrak{L}^{p}$. This is achieved in two steps. Our main technical result is the following theorem (Theorem 4.1 of the main text).

Theorem 1.1. Let $\mathfrak{L}^{p}$ and $\mathfrak{L}^{q}$ be two Schatten ideals, $1 \leqslant p<q<\infty$. Then the inclusion $\mathfrak{L}^{p} \rightarrow \mathfrak{L}^{q}$ induces an invertible element in the bivariant entire cyclic homology $\mathrm{HE}_{0}\left(\mathfrak{L}^{p}, \mathfrak{L}^{q}\right)$. Consequently, the entire cyclic homology and cohomology of the two algebras are isomorphic:

$$
\operatorname{HE}^{i}\left(\mathfrak{L}^{p}\right)=\operatorname{HE}^{i}\left(\mathfrak{L}^{q}\right) ; \quad \operatorname{HE}_{i}\left(\mathfrak{L}^{p}\right)=\operatorname{HE}_{i}\left(\mathfrak{L}^{q}\right)
$$

for $i=0,1$.

Our proof follows the strategy employed by Cuntz in the proof of a similar result in the context of the algebraic periodic cyclic cohomology and $k k$-theory [7]. To make the proof work we need to establish certain key algebraic properties of the bivariant entire cyclic cohomology, and this is the main technical difficulty treated in the paper. We use in a significant way the general theory of the entire cyclic cohomology for bornological algebras developed by Meyer [17]. In particular, we rely on the excision property satisfied by this theory.

Thus all Schatten ideals $\mathfrak{L}^{p}$ have the same entire cyclic homology and cohomology, and so it is sufficient to perform the explicit computation for one of them. This is easiest to do for the trace class operators $\mathfrak{L}^{1}$ and indeed Meyer proves $[\mathbf{1 7}$, Thm 3.41] that $\operatorname{HE}^{i}\left(\mathfrak{L}^{1}\right)=\operatorname{HE}^{i}(\mathbb{C})$ and $\operatorname{HE}_{i}\left(\mathfrak{L}^{1}\right)=\operatorname{HE}_{i}(\mathbb{C})$ for $i=0,1$. We provide a different argument in the case of cohomology which is based on the properties of Hochschild cohomology of $\mathfrak{L}^{1}$. Combining this result with our first theorem we have (Theorem 5.2):

Received September 23, 2004, revised March 18, 2005; published on November 12, 2005. 2000 Mathematics Subject Classification: 19D55.

Key words and phrases: Bivariant entire cyclic cohomology, bornological algebras, Schatten ideals, p-summable Fredholm modules.

(C) 2005, Jacek Brodzki and Roger Plymen. Permission to copy for private use granted. 
Theorem 1.2. For any $p, 1 \leqslant p<\infty$,

$$
\operatorname{HE}^{0}\left(\mathfrak{L}^{p}\right)=\mathbb{C}, \quad \operatorname{HE}^{1}\left(\mathfrak{L}^{p}\right)=0
$$

and

$$
\operatorname{HE}_{0}\left(\mathfrak{L}^{p}\right)=\mathbb{C}, \quad \operatorname{HE}_{1}\left(\mathfrak{L}^{p}\right)=0 .
$$

This result has a very interesting application to the study of characters of $p$ summable Fredholm modules, which we treat in the last section. As is well known, an even $p$-summable Fredholm module $\alpha_{\gamma}$ over an algebra $A$ gives rise to a periodic cyclic cohomology class $\operatorname{ch}_{P}\left(\alpha_{\gamma}\right) \in H P^{0}(A)$. This class is the image of the class in $\operatorname{HP}^{0}\left(\mathfrak{L}^{p}\right)$ under the Chern character map

$$
\operatorname{ch}_{P}: \operatorname{HP}^{0}\left(\mathfrak{L}^{p}\right) \rightarrow \operatorname{HP}^{0}(A)
$$

Exactly the same procedure works in the entire cyclic cohomology and produces an entire cyclic cohomology class $\operatorname{ch}_{E}\left(\alpha_{\gamma}\right)$ in $H E^{0}(A)$. Again, this class is the image of a class in $\operatorname{HE}\left(\mathfrak{L}^{p}\right)$ under the Chern character map

$$
\operatorname{ch}_{E}: \operatorname{HE}^{0}\left(\mathfrak{L}^{p}\right) \rightarrow \operatorname{HE}^{0}(A)
$$

The two constructions are compatible in the sense that there is a commutative diagram

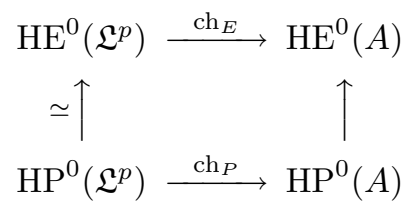

Here the vertical arrows are given by the canonical inclusion $\operatorname{HP}^{i}(\cdot) \rightarrow \operatorname{HE}^{i}(\cdot)$, $i=0,1$. We derive two conclusions from this construction. First, the classes $\operatorname{ch}_{P}\left(\alpha_{\gamma}\right)$ and $\operatorname{ch}_{E}\left(\alpha_{\gamma}\right)$ are both images of the generator of $\operatorname{HP}^{0}\left(\mathfrak{L}^{p}\right)=\mathbb{C}$. Secondly, the vertical map on the right maps the class $\operatorname{ch}_{P}\left(\alpha_{\gamma}\right)$ to $\operatorname{ch}_{E}\left(\alpha_{\gamma}\right)$. This implies, in particular, that the entire cocycle representing the class $\operatorname{ch}_{E}\left(\alpha_{\gamma}\right)$ is cohomologous to the periodic cocycle representing $\operatorname{ch}_{P}\left(\alpha_{\gamma}\right)$. This provides a simple conceptual framework for the transgression result of Connes-Moscovici [5] in the case of bounded Fredholm modules. The bivariant version of the Connes-Moscovici transgression has been developed by Perrot in [19]. We treat the case of both the even and odd Fredholm modules.

We conclude by mentioning the paper by Mathai and Stevenson [16] where our Theorems 1.1 and 1.2 have found an interesting geometric application.

\section{Spaces with bornology}

A bornology on a set $X$ is a family $\mathcal{B}$ of subsets of $X$ which is stable under the formation of subsets and finite unions $[\mathbf{1}]$. Elements of $\mathcal{B}$ will be called bounded sets. A base of a bornology $\mathcal{B}$ is a subfamily $\mathcal{B}_{0}$ of $\mathcal{B}$ with the property that any element of $\mathcal{B}$ is contained in some element of $\mathcal{B}_{0}$. A map $f:(X, \mathcal{B}) \rightarrow\left(Y, \mathcal{B}^{\prime}\right)$ of bornological spaces is bounded if and only if for any $B \in \mathcal{B}, f(B) \in \mathcal{B}^{\prime}$. 
A bornology on a vector space $E$ is said to be compatible with the vector space structure iff the vector addition $E \times E \rightarrow E$ and multiplication by scalars $\mathbb{C} \times E \rightarrow E$ are bounded maps of bornological spaces.

When the vector space $E$ is equipped with a topology, e.g. $E$ is a locally convex topological vector space, then there is a canonical bornology associated with the topology consisting of all sets which are absorbed by all neighbourhoods of zero. This is the so called von Neumann bornology. However, one can equip the vector space $E$ with a bornology which does not arise from the topology of $E$. One consequence of this is that the class of bounded linear maps on $E$ will be different in general from the class of continuous maps.

The vector space $E$ is called a convex bornological space if it is equipped with a bornology whose base consists of convex sets. In this case the base can be assumed to consist of balanced convex sets, which will be called discs. If $D$ is a bounded disc in $E$ we denote by $E_{D}$ the vector space generated by $D$ and equipped with the seminorm given by the gauge of $D$. When $E$ is a Hausdorff space, $E_{D}$ is a normed space. The spaces $E_{D}$ form an inductive system indexed by the directed family of bounded discs and $E$ is the direct limit of this system.

We shall say that a disc $D$ is pre-complete iff the space $E_{D}$ is complete. Thus when $E$ is Hausdorff, $E_{D}$ is a Banach space. We shall say that $E$ is a complete bornological space iff its bornology admits a base consisting of pre-complete discs (cf. [13, Def. IV.2.1]). A bornological space $E$ is complete iff it is the inductive limit of an injective inductive system of Banach spaces [13, IV.2.3] [17, Theorem A.4]. Every bornological space $E$ admits a bornological completion, but this operation is less well behaved than the usual completion of a uniform space (see [13, Chapter 4] [17, Appendix A] for a full discussion).

We say that $A$ is a bornological algebra iff it is equipped with a vector space bornology with respect to which the product map $A \times A \rightarrow A$ is bounded. A complete bornological algebra is one that is complete as a bornological vector space.

The construction of cyclic type homology theories requires the use of tensor products of algebras. In the study of cohomology theories like the entire cyclic cohomology of Banach algebras it is important to have control over bounded sets in the tensor product of algebras. However, as is well known from the work of Grothendieck [11, Probléme des Topologies, p. 33], there is in general no obvious relation between bounded sets in the tensor product and bounded sets in the algebra. A possible resolution of this problem, which works well in some situations, is to define completed tensor products with respect to a given bornology rather than topology.

The bornological tensor product of two bornological spaces $\left(E_{1}, \mathcal{B}_{1}\right)$ and $\left(E_{2}, \mathcal{B}_{2}\right)$ is by definition the algebraic tensor product $E_{1} \otimes E_{2}$ equipped with the bornology whose base consists of balanced convex hulls of sets of the form $B_{1} \otimes B_{2}$, where $B_{1} \in \mathcal{B}_{1}$ and $B_{2} \in \mathcal{B}_{2}$. The completed bornological tensor product $E_{1} \hat{\otimes} E_{2}$ is by definition the bornological completion of $E_{1} \otimes E_{2}$ with respect this bornology.

For example, the completed bornological tensor product of two Fréchet spaces equipped with the precompact bornology is isomorphic to the completed projective tensor product of the two spaces [17, Theorem 2.29]. 
When $V_{1}$ and $V_{2}$ are nuclear $L F$-spaces regarded as bornological spaces with the von Neumann bornology then the completed bornological tensor product $V_{1} \hat{\otimes} V_{2}$ is isomorphic to the inductive tensor product $V_{1} \bar{\otimes} V_{2}$ of Grothendieck, see $[\mathbf{1 7}$, Cor. 2.30, p. 15].

\section{The $X$-complex}

All cyclic type homology theories of an algebra $A$ are defined using a $\mathbb{Z} / 2 \mathbb{Z}$-graded complex associated with $A$ which, loosely speaking, is constructed using a certain deformation of the tensor algebra of $A$. We describe this construction first in the case of an algebra $A$ without any topology or bornology.

We recall the differential graded algebra of differential forms $\Omega A$ associated with $A$. By definition, $\Omega A$ is generated by elements of $A$ together with symbols $d a$, for $a$ in $A$, such that $d a$ is linear in $a$ and satisfies the Leibniz rule $d(a b)=(d a) b+a d b$. If the algebra $A$ is unital with unit 1 , it is not assumed that $d(1)=0$. As a consequence, in degree $n, \Omega^{n} A=A^{\otimes n+1} \oplus A^{\otimes n}$. Elements of $\Omega^{n} A$ are linear combinations of differential forms $a_{0} d a_{1} \ldots d a_{n}$ and $d a_{1} \ldots d a_{n}$, with $a_{i}$ in $A$. The graded space $\Omega A$ is turned into a differential complex by means of two operators

$$
\mathbf{b}=\left(\begin{array}{cc}
b & 1-\lambda \\
0 & -b^{\prime}
\end{array}\right), \quad \mathrm{B}=\left(\begin{array}{cc}
0 & 0 \\
N & 0
\end{array}\right)
$$

where the operators $b^{\prime}, b, \lambda, N$ have their usual meaning, c.f. [7, p.9].

For any algebra $A$ we define the $X$-complex $X(A)$ of $A$ to be the $\mathbb{Z} / 2 \mathbb{Z}$-graded complex

$$
A \underset{b}{\stackrel{\natural d}{\rightleftarrows}} \Omega^{1} A_{\natural}
$$

where $\Omega^{1} A_{\natural}=\Omega^{1} A /\left[A, \Omega^{1} A\right]=\Omega^{1} A / b\left(\Omega^{2} A\right)$, and $\natural: \Omega^{1} A \rightarrow \Omega^{1} A_{\natural}$ is the canonical projection map, compare $[\mathbf{7}$, p. 21].

In order to obtain an interesting homology theory we need to apply the above construction to the non-unital tensor algebra $T A$ of the algebra $A$. While it seems at first sight that this will lead to a huge and unwieldy object, it turns out in fact, thanks to the following result [9, Theorem 5.5], that the resulting complex is a deformation of the mixed complex $(\Omega A, \mathrm{~b}, \mathrm{~B})$. A starting point for the proof is a remark that the tensor algebra of any algebra $A$ can be identified with the even part of the algebra $\Omega A$ equipped with the Fedosov product

$$
\omega \circ \eta=\omega \eta-(-1)^{\operatorname{deg} \omega} d \omega d \eta .
$$

Proposition 3.1. ([9][7, Thm. 2.29, p. 24]) For any algebra $A$ the $X$-complex of the tensor algebra $T A$ of $A$ is isomorphic to a complex of the form

$$
\Omega^{e v} A \underset{\beta}{\stackrel{\delta}{\rightleftarrows}} \Omega^{o d d} A
$$

where the differentials $\beta$ and $\delta$ can be explicitly determined in terms of differentials $\mathrm{b}$ and $\mathrm{B}$. 
Let us assume that $A$ is a complete bornological algebra. Then $\Omega A$ becomes a bornological algebra with bornology whose base is given by balanced convex hulls of the sets $S d S \ldots d S$ and $d S \ldots d S$, where $S$ is an element of the bornology on $A$. We denote by $\Omega_{\mathrm{an}} A$ the completion of $\Omega A$ with respect to this bornology. This is a $\mathbb{Z} / 2 \mathbb{Z}$-graded complex with the same differentials $\mathrm{b}$ and $\mathrm{B}$, which are now bounded maps. The even part of the algebra $\Omega_{\mathrm{an}} A$, equipped with the Fedosov product, is by definition the analytic tensor algebra $\mathcal{T} A$ of $A$. This algebra fits into the algebra extension

$$
0 \rightarrow \mathfrak{J} A \rightarrow \mathcal{T} A \rightarrow A \rightarrow 0
$$

The $X$-complex of the tensor algebra $\mathcal{T} A$ is defined in the same way as before.

Definition 3.2. The bivariant entire cyclic homology of a pair of bornological algebras $A$ and $B$ is by definition

$$
\operatorname{HE}_{*}(A, B)=H_{*}(\operatorname{Hom}(X(\mathcal{T} A), X(\mathcal{T} B))
$$

where $\operatorname{Hom}(X(\mathcal{T} A), X(\mathcal{T} B))$ denotes the $\mathbb{Z} / 2 \mathbb{Z}$-grade complex of bounded linear maps from $X(\mathcal{T} A)$ to $X(\mathcal{T} B)$. This complex is equipped with the differential $[\partial, \phi]=$ $\partial \circ \phi-(-1)^{\operatorname{deg} \phi} \phi \circ \partial$, where $\partial=\mathrm{b}+\mathrm{B}[\mathbf{7}$, p. 57] [17, p. 37]. The resulting homology theory is $\mathbb{Z} / 2 \mathbb{Z}$-graded.

This construction is due to Meyer, who proves that this bivariant cyclic homology satisfies excision in both variables $[\mathbf{1 7}][\mathbf{7}$, Thm. 5.4]. More precisely, we have the following.

Theorem 3.3. Let $0 \rightarrow S \rightarrow P \rightarrow Q \rightarrow 0$ be an extension of complete bornological algebras which admits a bounded linear section. Assume further that $A$ is a complete bornological algebra. Then we have the following natural exact sequences of length six.

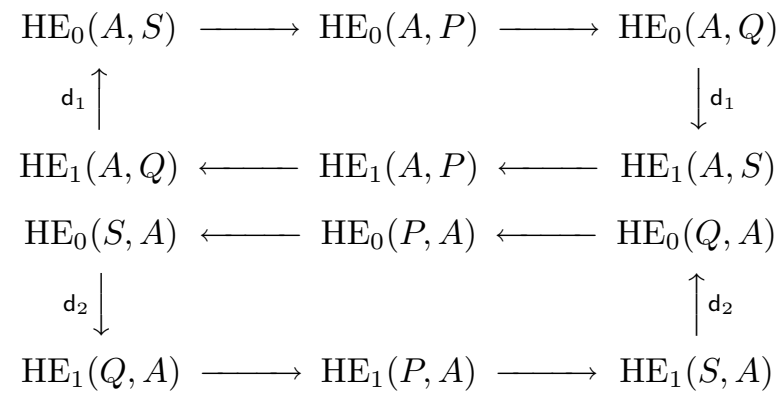

Moreover, HE is invariant with respect to differentiable homotopies whose first derivative is integrable. Meyer also proves that when $A$ is a Banach algebra then $\operatorname{HE}_{*}(A, \mathbb{C})$ is the same as the entire cyclic cohomology $\operatorname{HE}^{*}(A)$ of $A$ as defined by Connes $[\mathbf{1 8}, 4.1]$

A very important property of HE is the existence of the composition product, which is defined as in the case of bivariant periodic cyclic homology by composition of linear chain maps. For any three bornological algebras $A_{1}, A_{2}$ and $A_{3}$ there is a bilinear product:

$$
\operatorname{HE}_{i}\left(A_{1}, A_{2}\right) \times \operatorname{HE}_{j}\left(A_{2}, A_{3}\right) \rightarrow \operatorname{HE}_{i+j}\left(A_{1}, A_{3}\right)
$$


given by $f \cdot g=g \circ f$. A detailed study of this product will provide formulae for the connecting homomorphisms in the exact sequences of Theorem 3.3.

\section{Entire cyclic cohomology of Schatten ideals}

The aim of this section is the following

Theorem 4.1. Let $\mathfrak{L}^{p}$ and $\mathfrak{L}^{q}$ be two Schatten ideals, $1 \leqslant p<q$. Then the inclusion $\mathfrak{L}^{p} \rightarrow \mathfrak{L}^{q}$ induces an invertible element in $\operatorname{HE}_{0}\left(\mathfrak{L}^{p}, \mathfrak{L}^{q}\right)$. Consequently, the entire cyclic homology and cohomology of the two algebras are isomorphic:

$$
\operatorname{HE}^{i}\left(\mathfrak{L}^{p}\right)=\operatorname{HE}^{i}\left(\mathfrak{L}^{q}\right) ; \quad \operatorname{HE}_{i}\left(\mathfrak{L}^{p}\right)=\operatorname{HE}_{i}\left(\mathfrak{L}^{q}\right)
$$

for $i=0,1$.

In the context of algebraic periodic cyclic homology [7] and in $k k$-theory this result was first proved by Cuntz [6]. The proof outlined here follows the same strategy, which relies on algebraic features of bivariant cohomology theories. To make sure that this translation works, we need to prove a number of technical results which provide the necessary formal properties of the bivariant entire cyclic homology.

Let $E$ denote the following extension of complete bornological algebras.

$$
E: \quad 0 \rightarrow S \stackrel{i}{\rightarrow} P \stackrel{p}{\rightarrow} Q \rightarrow 0
$$

We shall assume that this sequence is split, i.e. there exists a bounded linear map $s: Q \rightarrow P$ which is a right inverse for the projection $p$.

The excision property of the bivariant cyclic homology $\mathrm{HE}_{*}$ implies that there are the following two exact sequences

$$
\begin{aligned}
& \rightarrow \operatorname{HE}_{*}(P, S) \rightarrow \operatorname{HE}_{*}(S, S) \stackrel{\delta_{1}}{\longrightarrow} \operatorname{HE}_{*+1}(Q, S) \rightarrow \\
& \rightarrow \operatorname{HE}_{*}(Q, P) \rightarrow \operatorname{HE}_{*}(Q, Q) \stackrel{\delta_{2}}{\longrightarrow} \operatorname{HE}_{*+1}(Q, S) \rightarrow
\end{aligned}
$$

Denote by $1_{Q}$ the class in $\operatorname{HE}_{0}(Q, Q)$ induced by the identity map on the algebra $Q$ and, similarly, $1_{S}$ will denote the class of the identity map on $S$. The following lemma is a translation of a result of Kassel [14, Lemme 2.2] in the case of his bivariant cyclic cohomology. An analogous result has been proved in the case of bivariant periodic cyclic cohomology by Cuntz and Quillen [10][7, Prop. 2.51, p.33].

Proposition 4.2. If $\delta_{1}$ and $\delta_{2}$ denote the connecting homomorphisms in the preceding two long exact sequences, then

$$
\delta_{1}\left(1_{S}\right)=-\delta_{2}\left(1_{Q}\right) \in \operatorname{HE}_{1}(Q, S)
$$

Proof. To simplify notation, for any two $\mathbb{Z} / 2 \mathbb{Z}$-graded complexes $C$ and $D$, we shall write $\mathrm{H}_{*}(C, D)$ for the homology $\mathrm{H}_{*}(\operatorname{Hom}(C, D))$.

The projection $p$ induces a map of complexes $X(\mathcal{T} P) \rightarrow X(\mathcal{T} Q)$. Let us denote by $X(P, Q)$ the kernel of this map, so that we have an exact sequence of $\mathbb{Z} / 2 \mathbb{Z}$ graded complexes

$$
(\alpha): \quad 0 \rightarrow X(P, Q) \rightarrow X(\mathcal{T} P) \rightarrow X(\mathcal{T} Q) \rightarrow 0
$$


By the universal properties of the non-unital tensor algebra and the $X$-complex, a linear splitting $s$ of the sequence $E$ induces a linear splitting of the sequence $(\alpha)$ $[\mathbf{1 7}, 3.3 .2]$ which will also be denoted $s$. This splitting in turn determines the odd degree map $[\partial, s]=\partial \circ s-s \circ \partial$ with the properties $p[\partial, s]=0$ and $[\partial,[\partial, s]]=0$. Thus $[\partial, s]$ determines an element in $\mathrm{H}_{1}(X(\mathcal{T} Q), X(P, Q))$ which will be denoted $\gamma$. This class does not depend on the choice of the linear section $s$. Indeed, any two $\mathbb{C}$-splittings can be connected by a linear path $(1-t) s+t s^{\prime}$. Now:

$$
\left[\partial,(1-t) s+t s^{\prime}\right]=(1-t)[\partial, s]+t\left[\partial, s^{\prime}\right]
$$

gives a (differentiable) homotopy between the corresponding cycles.

Furthermore, there is the induced sequence of $\mathbb{Z} / 2 \mathbb{Z}$-graded complexes

$$
\begin{aligned}
0 \rightarrow & \operatorname{Hom}(X(\mathcal{T} Q), X(P, Q)) \rightarrow \operatorname{Hom}(X(\mathcal{T} P), X(P, Q)) \\
& \rightarrow \operatorname{Hom}(X(P, Q), X(P, Q)) \rightarrow 0
\end{aligned}
$$

If we now apply the homology functor we obtain an exact sequence of length six. The two connecting homomorphisms

$$
\mathrm{H}_{j}(X(P, Q), X(P, Q)) \stackrel{\gamma \cdot}{\longrightarrow} \mathrm{H}_{j+1}(X(\mathcal{T} Q), X(P, Q)),
$$

for $j=0,1$, in the resulting homology sequence are both given by multiplication by $\gamma$.

The inclusion map $i: X(\mathcal{T} S) \rightarrow X(\mathcal{T} P)$ satisfies $p i=0$ and so can be regarded as a map $i: X(\mathcal{T} S) \rightarrow X(P, Q)$. Since this map is induced from an algebra homomorphism, it is a 0 -cycle and so creates an element $i \in \mathrm{H}_{0}(X(\mathcal{T} S), X(P, Q))$. A key step in the proof of excision in HE (Theorem 3.3) is the fact that $i$ is invertible [17], so that there exists $i^{-1} \in \mathrm{H}_{0}(X(P, Q), X(\mathcal{T} S))$.

For any $\mathbb{Z} / 2 \mathbb{Z}$-graded complex $C$ the composition product gives a map

$$
\mathrm{H}_{j}(X(\mathcal{T} S), X(P, Q)) \otimes \mathrm{H}_{k}(X(P, Q), C) \rightarrow \mathrm{H}_{j+k}(X(\mathcal{T} S), C) .
$$

Thus taking the product on the left by the invertible element $i$ of degree 0 establishes an isomorphism

$$
i \cdot: \mathrm{H}_{j}(X(P, Q), C) \stackrel{\simeq}{\longrightarrow} \mathrm{H}_{j}(X(\mathcal{T} S), C) .
$$

If we now recall that $\operatorname{HE}_{*}(S, S)=\mathrm{H}_{*}(X(\mathcal{T} S), X(\mathcal{T} S))$ and use the triple product

$$
\begin{aligned}
\mathrm{H}_{0}(X(P, Q), X(\mathcal{T} S)) \otimes & \mathrm{H}_{j}(X(\mathcal{T} S), X(\mathcal{T} S)) \otimes \mathrm{H}_{0}(X(\mathcal{T} S), X(P, Q)) \\
& \longrightarrow \mathrm{H}_{j}(X(P, Q), X(P, Q))
\end{aligned}
$$

we deduce that there is an isomorphism

$$
\operatorname{HE}_{j}(S, S) \simeq \mathrm{H}_{j}(X(P, Q), X(P, Q)) .
$$

For $\phi \in \operatorname{HE}_{*}(S, S)$ this is given explicitly by $\phi \mapsto i^{-1} \cdot \phi \cdot i$. Taking the left product with $\gamma$ produces a map

$$
\operatorname{HE}_{j}(S, S) \rightarrow \mathrm{H}_{j+1}(X(\mathcal{T} Q), X(P, Q)), \quad \phi \mapsto \gamma \cdot i^{-1} \cdot \phi \cdot i .
$$

If we now take the product on the right with $i^{-1} \in \mathrm{H}_{0}(X(P, Q), X(\mathcal{T} S))$ and use the identification $\mathrm{H}_{*}(X(\mathcal{T} Q), X(\mathcal{T} S))=\operatorname{HE}_{*}(Q, S)$ we conclude that the connecting 
homomorphism $\operatorname{HE}_{j}(S, S) \rightarrow \operatorname{HE}_{j+1}(Q, S)$ may be described by the fomula

$$
\phi \mapsto \gamma \cdot i^{-1} \cdot \phi,
$$

for any $\phi \in \operatorname{HE}_{j}(S, S)$. In particular, when $\phi=1_{S}$ we obtain

$$
\delta_{1}\left(1_{S}\right)=\gamma \cdot i^{-1}
$$

The exact sequence $(\alpha)$ also leads to the following exact sequence:

$$
\begin{aligned}
0 \rightarrow & \operatorname{Hom}(X(\mathcal{T} Q), X(P, Q)) \rightarrow \operatorname{Hom}(X(\mathcal{T} Q), X(\mathcal{T} P)) \\
& \rightarrow \operatorname{Hom}(X(\mathcal{T} Q), X(\mathcal{T} Q)) \rightarrow 0
\end{aligned}
$$

which in turn induces a corresponding exact sequence of bivariant homology groups of length six.

The existence of a bounded linear splitting $s$ of the sequence $(\alpha)$ implies that $X(\mathcal{T} P)$ splits as a direct sum of $\mathbb{Z} / 2 \mathbb{Z}$-graded vector spaces:

$$
X(\mathcal{T} P)=X(\mathcal{T} Q) \oplus X(P, Q)
$$

It then follows that there is a complementary splitting of the sequence $(\alpha)$ given by $s^{\prime}=1-s$. It is now not difficult to see that the two connecting homomorphisms

$$
\mathrm{H}_{j}(X(\mathcal{T} Q), X(\mathcal{T} Q)) \rightarrow \mathrm{H}_{j+1}(X(\mathcal{T} Q), X(P, Q))
$$

for $j=0,1$ are given by left multiplication by the element $\left[\partial, s^{\prime}\right]=-[\partial, s]=-\gamma$.

We can find an explicit formula for the connecting homomorphism

$$
\delta_{2}: \operatorname{HE}_{j}(Q, Q) \rightarrow \operatorname{HE}_{j+1}(Q, S)
$$

as follows. We multiply on the left by $-\gamma$ to construct a map

$$
\operatorname{HE}_{j}(Q, Q):=\mathrm{H}_{j}(X(\mathcal{T} Q), X(\mathcal{T} Q)) \stackrel{-\gamma \cdot}{\longrightarrow} \mathrm{H}_{j+1}(X(\mathcal{T} Q), X(P, Q)) .
$$

Multiplication on the right by $i^{-1} \in \mathrm{H}_{0}(X(P, Q), X(\mathcal{T} S))$ gives a map

$$
\mathrm{H}_{j+1}(X(\mathcal{T} Q), X(P, Q)) \stackrel{i^{-1}}{\longrightarrow} \mathrm{H}_{j+1}(X(\mathcal{T} Q), X(\mathcal{T} S))=\operatorname{HE}_{j+1}(Q, S) .
$$

Thus the formula for the connecting homomorphism $\delta_{2}$ is

$$
\delta_{2}(\psi)=-\gamma \cdot \psi \cdot i^{-1}
$$

for any $\psi \in \operatorname{HE}_{j}(Q, Q)$. In particular, when $\psi=1_{Q}$ we have

$$
\delta_{2}\left(1_{Q}\right)=-\gamma \cdot i^{-1}=-\delta_{1}\left(1_{S}\right)
$$

This result can be extended to provide formulae for connecting homomorphisms in exact sequences of Theorem 3.3. Both of the excision exact sequences are natural. In the case of the sequence (1) this means that there exists a commutative diagram

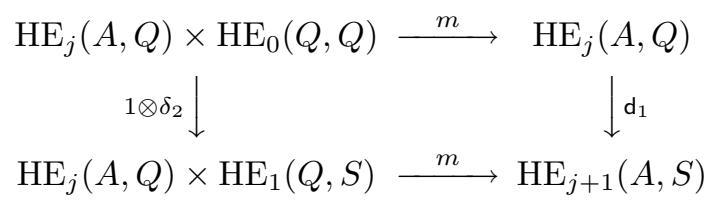


where $m$ denotes the product map and $d_{1}$ denotes the connecting homomorphism in the diagram (1) of Theorem 3.3 , for $j=0,1$.

Taking into account the usual sign convention we have that

$$
\mathrm{d}_{1}(\phi \cdot \psi)=m\left(1 \otimes \delta_{2}\right)(\phi \otimes \psi)=m\left((-1)^{\operatorname{deg}(\phi)}\left(\phi \otimes \delta_{2}(\psi)\right)=(-1)^{\operatorname{deg}(\phi)} \phi \cdot \delta_{2}(\psi)\right.
$$

for $\phi \in \operatorname{HE}_{j}(A, Q)$ and $\psi \in \operatorname{HE}_{0}(Q, Q)$. Hence

$$
\mathrm{d}_{1}(\phi)=\mathrm{d}_{1}\left(\phi \cdot 1_{Q}\right)=(-1)^{\operatorname{deg}(\phi)} \phi \cdot \delta_{2}\left(1_{Q}\right)
$$

Similarly, we obtain a formula for the connecting homomorphism $\mathrm{d}_{2}$ in the exact sequence (2). In this case the naturality of this sequence implies that there exists the following commutative diagram

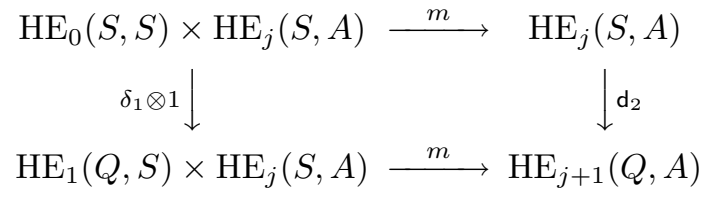

Hence, for $\phi \in \operatorname{HE}_{0}(S, S)$ and $\phi \in \operatorname{HE}_{j}(S, A)$ we have

$$
\mathrm{d}_{2}(\phi \cdot \psi)=m\left(\delta_{1} \otimes 1\right)(\phi \otimes \psi)=\delta_{1}(\phi) \cdot \psi
$$

Thus

$$
\mathrm{d}_{2}(\psi)=\mathrm{d}_{2}\left(1_{S} \cdot \psi\right)=\delta_{1}\left(1_{S}\right) \cdot \psi
$$

In summary, we have obtained the proof of the following proposition, which extends an analogous result of Kassel [14, Thm 2.1, Lemme 2.2] (see also [10, Thm $5.5])$.

Proposition 4.3. Let us denote by $\operatorname{ch}(E)$ the class $-\delta_{1}\left(1_{S}\right)=\delta_{2}\left(1_{Q}\right)$ of the extension $E$. Then the connecting homomorphism $\mathrm{d}_{1}$ in the exact sequence (1) sends $\phi \in \mathrm{HE}_{j}(A, Q)$ to $(-1)^{\operatorname{deg}(\phi)} \phi \cdot \operatorname{ch}(E) \in \mathrm{HE}_{j+1}(A, S)$. The connecting homomorphism $\mathrm{d}_{2}$ in the sequence (2) sends $\psi \in \operatorname{HE}_{j}(S, A)$ to $\operatorname{ch}(E) \cdot \psi \in \operatorname{HE}_{j+1}(Q, A)$.

This implies, as in $[\mathbf{1 4}][\mathbf{1 0}]$, the following.

Corollary 4.4. If the algebra $P$ in the extension $E$ is HE-equivalent to 0 , which means that $\mathrm{HE}_{*}(A, P)=\mathrm{HE}_{*}(P, A)=0$ for any bornological algebra $A$, then $\operatorname{ch}(E)$ is an invertible element in $\mathrm{HE}_{1}(Q, S)$.

Proof. Let us put $A=S$ in the sequence (1) and then $A=Q$ in the sequence (2) of Theorem 3.3. Since the terms containing the algebra $P$ are zero, we see that the connecting homomorphisms $\mathrm{d}_{1}$ and $\mathrm{d}_{2}$ are now isomorphisms. In particular, there exists $\eta_{1} \in \operatorname{HE}_{1}(S, Q)$ such that $\mathrm{d}_{1}\left(\eta_{1}\right)=1_{S} \in \mathrm{HE}_{0}(S, S)$. Similarly, there exists $\eta_{2} \in \operatorname{HE}_{1}(S, Q)$ such that $\mathrm{d}_{2}\left(\eta_{2}\right)=1_{Q} \in \operatorname{HE}_{0}(Q, Q)$. But we have just established that

$$
\mathrm{d}_{1}\left(\eta_{1}\right)=\eta_{1} \cdot \operatorname{ch}(E)=1_{S}
$$

and that

$$
\mathrm{d}_{2}\left(\eta_{2}\right)=\operatorname{ch}(E) \cdot \eta_{2}=1_{Q}
$$


This implies that $\eta_{1}=\eta_{2}$. Indeed,

$$
\eta_{1}=\eta_{1} \cdot 1_{Q}=\eta_{1} \cdot \operatorname{ch}(E) \cdot \eta_{2}=1_{S} \cdot \eta_{2}=\eta_{2}
$$

Thus $\eta=\eta_{1}=\eta_{2} \in \operatorname{HE}_{1}(S, Q)$ is the inverse of $\operatorname{ch}(E) \in \operatorname{HE}_{1}(Q, S)$.

The rest of the proof of the theorem follows an argument of Cuntz [6, Satz 6.12][7], who used it to prove an analogous result in his $k k$ theory and periodic cyclic homology.

Proposition 4.5. Let us assume that for two complete bornological algebras $A$ and $B$ there are maps

$$
\begin{array}{ll}
\alpha: & B \hookrightarrow A \\
\beta: & A \otimes A \rightarrow B
\end{array}
$$

such that the composition $\alpha \circ \beta$ identical to the product map on $A$, whereas $\beta \circ \alpha \otimes \alpha$ is the product on $B$. Then the element $[\alpha]$ of $\operatorname{HE}_{0}(B, A)$ is invertible. This implies that $\operatorname{HE}^{*}(A) \simeq \operatorname{HE}^{*}(B)$ and $\operatorname{HE}_{*}(B) \simeq \operatorname{HE}_{*}(A)$.

Proof. We present here a more explicit version of Cuntz's argument, which is adapted to the context of entire cyclic homology.

We equip the Fréchet algebra $C^{\infty}([0,1])$ of smooth functions with the von Neumann bornology; in the present case this bornology coincides with the pre-compact bornology.

If $A$ is a complete bornological algebra, define

$$
A[0,1]=C^{\infty}([0,1]) \hat{\otimes} A
$$

We denote by $A(0,1]$ the algebra of smooth functions from the closed interval $[0,1]$ to $A$ which vanish at zero; we use the notation $A(0,1), A[0,1)$ to denote the algebras of smooth functions from the interval $[0,1]$ to $A$ that vanish at both ends of the interval or just at 1 . The algebra $A[0,1)$ is contractible to zero: the family of maps $\phi_{t}$ that send a function $f$ to $\phi_{t}(f)(x)=f((1-t)(x))$ forms a homotopy between the identity map and evaluation at 1 (which is the same as the zero map).

There is the following suspension extension:

$$
\mathfrak{S}(A): 0 \rightarrow A(0,1) \rightarrow A[0,1) \rightarrow A \rightarrow 0
$$

where the map on the right is given by evaluation at 0 . Since the algebra $A[0,1)$ is contractible it is HE-equivalent to zero. We can therefore use Corollary 4.4 to deduce that the class $\operatorname{ch} \mathfrak{S}(A)=-\delta_{1}\left(1_{A(0,1)}\right)=\delta_{2}\left(1_{A}\right) \in \operatorname{HE}_{1}(A, A(0,1))$ is invertible, for any complete bornological algebra $A$.

Let $A$ and $B$ be complete bornological algebras as in the statement of the Proposition. We denote by $\mathcal{B}$ the complete bornological algebra generated by the algebra $B(0,1)$ together with the algebra $A t=\left\{f_{a} \mid a \in A\right\}$ consisting of functions $f_{a}:[0,1] \rightarrow A$ which for a fixed $a \in A$ send $t \mapsto t a$. As a vector space, $\mathcal{B}$ is the direct sum of the two algebras. The product on $\mathcal{B}$ is defined using the pointwise product on $B(0,1)$ together with the following two operations. The product of a function $f \in B(0,1)$ by an element $g_{a} \in A t$ is given by $\mu\left(\alpha(f) \otimes g_{a}\right)$. Finally, the product of two fucntions $f_{a}$ and $f_{b}$ is the function $g(a, b)+f_{\alpha \mu(a \otimes b)}$ where

$$
g(a, b)(t)=\mu(a \otimes b)\left(t^{2}-t\right) .
$$


With these definitions we have the following extension of complete bornological algebras

$$
0 \rightarrow B(0,1) \rightarrow \mathcal{B} \rightarrow A \rightarrow 0
$$

which admits a bounded linear splitting. Proposition 4.2 implies that this extension creates an element $u \in \operatorname{HE}_{1}(A, B(0,1))$.

The homomorphism $\alpha: B \rightarrow A$ gives rise to an element $[\alpha] \in \operatorname{HE}_{0}(B, A)$. We need to show that it is invertible. For this we construct first the following diagram:

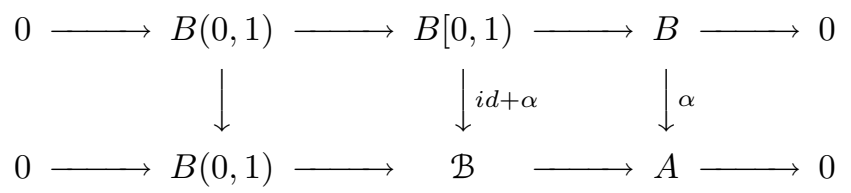

Using the first of the two excision sequences we obtain the following commutative diagram:

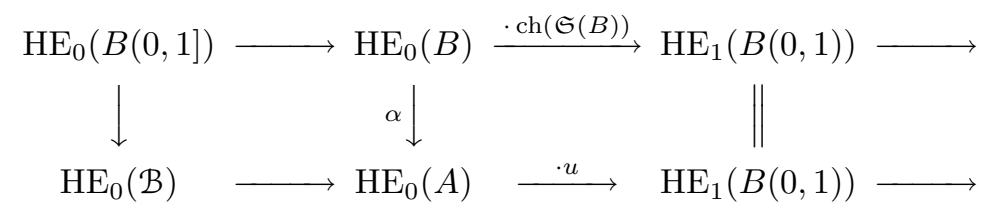

Here the two connecting homomorphisms on the right are in accordance with Proposition 4.3 whereas the vertical map in the middle is given by taking the product on the right with $\alpha \in \mathrm{HE}_{0}(B, A)$. Since the diagram commutes we see that for any $\phi \in \operatorname{HE}_{0}(B)$

$$
\phi \cdot \alpha \cdot u=\phi \cdot \operatorname{ch}(\mathfrak{S}(B))
$$

Given that $\operatorname{ch}(\mathfrak{S})$ is invertible we find that $\alpha \cdot u \cdot \operatorname{ch}(\mathfrak{S}(B))^{-1}=1 \in \operatorname{HE}_{0}(B, B)$.

We now employ the following commutative diagram:

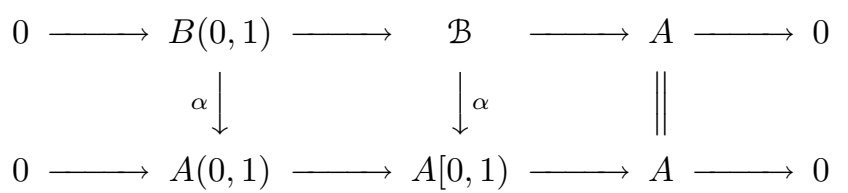

where the vertical map on the left is the obvious extension of $\alpha$ to functions. Using excision again, this translates to the following commutative diagram of homology groups:

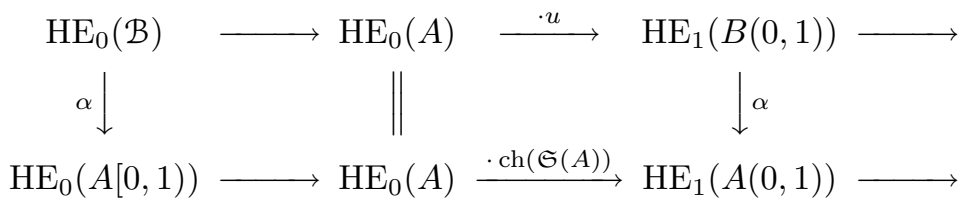

If we take into account the isomorphism of homology groups $\operatorname{HE}_{1}(B(0,1))=\mathrm{HE}_{0}(B)$ provided by the suspension extension (and similarly in the case of the bottom row) 
we obtain the commutative diagram:

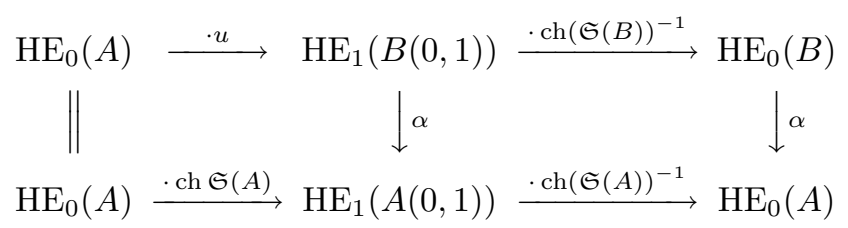

It is now clear that $u \cdot \operatorname{ch}(\mathfrak{S}(B))^{-1} \cdot \alpha=1 \in \operatorname{HE}_{0}(A, A)$. Thus $\alpha \in \operatorname{HE}_{0}(B, A)$ is invertible, with inverse $u \cdot \operatorname{ch}(B(0,1))^{-1} \in \operatorname{HE}_{0}(A, B)$.

To finish the proof of the theorem we let $B=\mathfrak{L}^{p}$ and $A=\mathfrak{L}^{q}$, where $p \leqslant q \leqslant 2 p$. The map $\alpha$ of the previous statements is obtained from the continuous inclusion $\mathfrak{L}^{p} \rightarrow \mathfrak{L}^{q}$ and the map $\beta$ from the multiplication map $\mathfrak{L}^{q} \hat{\otimes} \mathfrak{L}^{q} \rightarrow \mathfrak{L}^{p}$. This completes the proof of Theorem 4.1 .

Corollary 4.6. Let $\mathfrak{B}$ be a complete bornological algebra. Then for any $1 \leqslant p<q$ the inclusion $\mathfrak{L}^{p} \hat{\otimes} \mathfrak{B} \rightarrow \mathfrak{L}^{q} \hat{\otimes} \mathfrak{B}$ induces an invertible element in bivariant entire cyclic cohomology $\operatorname{HE}^{0}\left(\mathfrak{L}^{p} \hat{\otimes} \mathfrak{B}, \mathfrak{L}^{q} \hat{\otimes} \mathfrak{B}\right)$. Thus the entire cyclic homology and cohomology of the algebras $\mathfrak{L}^{p} \hat{\otimes} \mathfrak{B}$ and $\mathfrak{L}^{q} \hat{\otimes} \mathfrak{B}$ are isomorphic.

\section{Hochschild homology of $\mathfrak{L}^{1}$}

In the previous section we have established HE-equivalence of the Schatten ideals $\mathfrak{L}^{p}$ for all $p \geqslant 1$. To finish the computation we need to find explicit information about one of those ideals and it turns out that this is easiest to do in the case of the trace class operators. In the biviariant case this was treated by Meyer who proved the following stability result. [17, Thm 3.41]

Proposition 5.1. Let $\mathfrak{L}^{1}$ denote the ideal of trace class operators. Then

$$
\operatorname{HE}_{i}\left(\mathfrak{L}^{1}\right)=\operatorname{HE}_{i}(\mathbb{C}), \quad \operatorname{HE}^{i}\left(\mathfrak{L}^{1}\right)=\operatorname{HE}^{i}(\mathbb{C})
$$

for $i=0,1$.

It is interesting to provide a direct proof of this result in the case of cohomology. Let $E$ and $F$ be two Banach spaces in duality relative to a non-degenerate bilinear form $\langle-,-\rangle: E \times F \rightarrow \mathbb{C}$. Then the tensor product of these spaces can be turned into an algebra with the multiplication defined by

$$
(x \otimes y)\left(x^{\prime} \otimes y^{\prime}\right)=\left\langle x^{\prime}, y^{\prime}\right\rangle x \otimes y^{\prime}
$$

In the case where $E$ is a Hilbert space $H$ and $F$ is its continuous dual $H^{*}$, we have that $H \hat{\otimes} H^{*}=N(H)$, where $N(H)$ is the algebra of nuclear operators on $H$. When $H$ is separable, the algebra of nuclear operators is isomorphic to the algebra of trace class operators $\mathfrak{L}^{1}$.

Furthermore, Helemskii proves in [12, Ch. IV] that the algebra of nuclear operators $N(H)$, hence the algebra of trace class operators $\mathfrak{L}^{1}$ is biprojective, which means that it is a projective bimodule over itself. It is also proved in [12, Theorem V.2.28] that for a biprojective Banach algebra $A$ we have $H^{3}(A, X)=0$ for any 
Banach bimodule $X$. It then follows [15] that there exists a connection that provides a uniformly bounded contracting homotopy of the Hochschild complex for $A$ (with coefficients in $A$ ). This implies by the perturbation mapping lemma that the canonical inclusion $\operatorname{HP}^{*}(A) \rightarrow \operatorname{HE}^{*}(A)$ is an isomorphism [15]. On the left-hand side of this map, we regard $A$ as a topological algebra and define $\operatorname{HP}^{*}(A)$ via the projective tensor product.

To summarise, this sequence of arguments shows that $\operatorname{HP}^{*}\left(\mathfrak{L}^{1}\right) \simeq \operatorname{HE}^{*}\left(\mathfrak{L}^{1}\right)$. Finally, Cuntz proves in $[\mathbf{7}$, Prop. 17.3$]$ that $\operatorname{HP}^{*}\left(\mathfrak{L}^{1}\right)=\operatorname{HP}^{*}(\mathbb{C})$ and $\operatorname{HP}_{*}\left(\mathfrak{L}^{1}\right)=$ $\mathrm{HP}_{*}(\mathbb{C})$. We summarise these results as follows.

Theorem 5.2. The algebra $\mathfrak{L}^{1}$ is $\mathrm{HE}^{*}$-equivalent to $\mathbb{C}$.

In conclusion, we have the following.

Corollary 5.3. For any $p, 1 \leqslant p<\infty$,

$$
\operatorname{HE}^{0}\left(\mathfrak{L}^{p}\right)=\mathbb{C}, \quad \operatorname{HE}^{1}\left(\mathfrak{L}^{p}\right)=0
$$

and

$$
\operatorname{HE}_{0}\left(\mathfrak{L}^{p}\right)=\mathbb{C}, \quad \operatorname{HE}_{1}\left(\mathfrak{L}^{p}\right)=0 .
$$

Proof. This result follows immediately from Theorem 4.1 and Proposition 5.1.

\section{Canonical classes associated with $p$-summable Fredholm modules}

In this section we use our calculations to put some known results concerning characters of Fredholm modules in a new context. Let $A$ be an involutive algebra over $\mathbb{C}$.

We begin with the odd case. We recall that a bounded odd $p$-summable Fredholm module over $A$ is given by the data $(H, \pi, F)$, where $\pi: A \rightarrow \mathfrak{L}(H)$ is a representation the algebra $A$ on a Hilbert space $H$, and $F$ is a self-adjoint involution which commutes with $\pi$ modulo $\mathfrak{L}^{p}$ [4, p. 208].

Let $P$ be the corresponding spectral projection onto the +1 eigenspace. Let $\sigma: A \rightarrow \mathfrak{L}(H)$ be a linear map defined by $\sigma(a)=P \pi(a) P$ for all $a \in A$, where $\pi$ is the representation of $A$ as bounded operators on Hilbert space as required by the structure of a Fredholm module. The goal of this section is to construct canonical classes in the periodic and entire cohomology of the algebra A. Our construction relies on an idea of Cuntz and Quillen [8], and follows the method outlined by Cuntz in $[6,7]$.

Let $A^{\prime}=\mathfrak{L}^{p}+\sigma(A)$. This is a subalgebra of $\mathfrak{L}(H)$. The Schatten ideal $\mathfrak{L}^{p}$ is then an ideal in the algebra $A^{\prime}$ and we have the following short exact sequence of algebras, which is $\mathbb{C}$-split:

$$
0 \rightarrow \mathfrak{L}^{p} \rightarrow A^{\prime} \rightarrow A^{\prime} / \mathfrak{L}^{p} \rightarrow 0
$$

The linear map $\sigma$ can be viewed as a map $\sigma: A \rightarrow A^{\prime}$, which gives rise to an algebra homomorpism $\sigma: T A \rightarrow A^{\prime}$ which has the important property that it sends the 
canonical ideal $I A \subset T A$ to the Schatten class $\mathfrak{L}^{p}$. In other words, we have the following commutative diagram of short exact sequences:

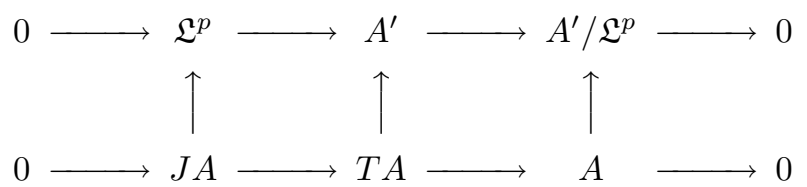

The algebra homomorphism $J A \rightarrow \mathfrak{L}^{p}$ gives rise to an element in $\operatorname{HP}_{0}\left(J A, \mathfrak{L}^{p}\right)=$ $\operatorname{HP}_{1}\left(A, \mathfrak{L}^{p}\right)$ and so to a map in cohomology $\operatorname{HP}^{*}\left(\mathfrak{L}^{p}\right) \rightarrow \operatorname{HP}^{*}(J A)=\operatorname{HP}^{*+1}(A)$. Given that $\operatorname{HP}^{0}\left(\mathfrak{L}^{p}\right)=\mathbb{C}$ and $\operatorname{HP}^{1}\left(\mathfrak{L}^{p}\right)=0$, an odd Fredholm module determines a canonical element in $\operatorname{HP}^{1}(A)$. This is the character of a $p$-summable Fredholm module that was first constructed by Connes in [2].

Our discussion of bornological algebras allows us to extend this idea to entire cyclic cohomology. Let $A$ be a complete bornological algebra. Applying the same reasoning as above to the canonical extension of complete bornological algebras

$$
0 \rightarrow \mathcal{J} A \rightarrow \mathcal{T} A \rightarrow A \rightarrow 0
$$

produces an element of $\operatorname{HE}_{1}\left(\mathcal{J} A, \mathfrak{L}^{p}\right)$ and so a map $\operatorname{HE}^{*}\left(\mathfrak{L}^{p}\right) \rightarrow \mathrm{HE}^{*}(\mathcal{J} A)$ $=\operatorname{HE}^{*+1}(A)$. Again, since we have that $\operatorname{HE}^{0}\left(\mathfrak{L}^{p}\right)=\mathbb{C}$ and $\operatorname{HE}^{1}\left(\mathfrak{L}^{p}\right)=0$, an odd $p$-summable Fredholm module determines a canonical class in $\operatorname{HE}^{1}(A)$. Furthermore, because of of HP and HE equivalence of Schatten ideals, these classes are independent of $1 \leqslant p<\infty$.

We remark that the canonical classes so constructed are compatible, in the sense that we have the following commutative diagram

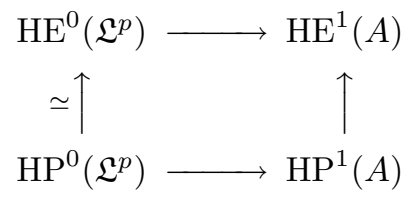

It is not difficult to work out the well-known explicit formulae for these characters; these were first derived by Connes in [2], compare [7, Ch. 19].

An even $p$-summable Fredholm module over an involutive $\mathbb{C}$ algebra $A$ is given by the data $(H, \pi, F, \gamma)$, where $\gamma$ is a self-adjoint involution on the Hilbert space $H$ (this Hilbert space is thus $\mathbb{Z} / 2 \mathbb{Z}$-graded) and the representation $\pi: A \rightarrow \mathfrak{L}(H)$ commutes with this involution (and so $A$ is represented by even operators with respect to the grading). $F$ and $\gamma$ anticommute and for each $a \in A,[F, \pi(a)] \in \mathfrak{L}^{p}$.

In this situation we have a different algebra extension:

$$
0 \rightarrow \mathfrak{L}^{p} \rightarrow A_{\gamma} \rightarrow A_{\gamma} / \mathfrak{L}^{p} \rightarrow 0
$$

where the algebra $A_{\gamma}$ is generated by $\mathfrak{L}^{p}$ and $\pi(A)$. This sequence has two linear splittings: $\pi$ and $\pi^{F}(a)=F \pi(a) F$, see [7, Ch. 19]. In the context on periodic cyclic cohomology this extension leads to the well known canonical character of Connes:

$$
\operatorname{HP}^{0}\left(\mathfrak{L}^{p}\right) \rightarrow \operatorname{HP}^{0}(A)
$$

which again is independent of $p$. This construction carries over to the case when the algebra $A$ is a complete bornological algebra; in particular the algebra extension (4) 
becomes an extension of complete bornological algebras. This extension gives rise to a map $\operatorname{HE}^{0}\left(\mathfrak{L}^{p}\right) \rightarrow \mathrm{HE}^{0}(A)$ which represents the character of an even Fredholm module. The two constructions, one in the context of periodic cyclic cohomology and the other for the entire cyclic cohomology, are compatible in the sense that there exists the following commutative diagram:

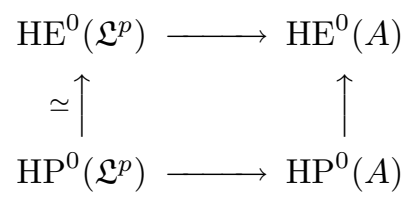

These remarks may be summarised as follows. Let $A$ be a complete involutive bornological algebra over $\mathbb{C}$. Let $\alpha$ be an odd $p$-summable Fredholm module over $A$, and let $\alpha_{\gamma}$ be an even $p$-summable Fredholm module over $A$. Let $\operatorname{ch}_{E}(\alpha) \in \operatorname{HE}^{1}(A)$ be the class in $\operatorname{HE}^{1}(A)$ determined by $\alpha$, and $\operatorname{ch}_{P}(\alpha) \in \operatorname{HP}^{1}(A)$ be the class in $\operatorname{HP}^{1}(A)$ determined by $\alpha$. Similarly, we denote by $\operatorname{ch}_{E}\left(\alpha_{\gamma}\right) \in \operatorname{HE}^{0}(A)$ and $\operatorname{ch}_{P}\left(\alpha_{\gamma}\right) \in \operatorname{HP}^{0}(A)$ the classes in $\operatorname{HE}^{0}(A)$ and $\operatorname{HP}^{0}(A)$ determined by $\alpha_{\gamma}$.

Theorem 6.1. Under the canonical inclusion

$$
\mathrm{HP}^{*}(A) \rightarrow \mathrm{HE}^{*}(A),
$$

$\operatorname{ch}_{E}(\alpha)$ is the image of $\operatorname{ch}_{P}(\alpha)$. In the even case, $\operatorname{ch}_{E}\left(\alpha_{\gamma}\right)$ is the image of $\operatorname{ch}_{P}\left(\alpha_{\gamma}\right)$.

Corollary 6.2. The class $\operatorname{ch}_{E}(\alpha) \in \operatorname{HE}^{1}(A)$ can be represented by a periodic cyclic cocycle. The class $\operatorname{ch}_{E}\left(\alpha_{\gamma}\right) \in \mathrm{HE}^{0}(A)$ can be represented by a periodic cyclic cocycle.

This means, in particular, that the entire cocycle representing the $\operatorname{class} \operatorname{ch}_{E}\left(\alpha_{\gamma}\right)$ is cohomologous to the periodic cocycle representing $\operatorname{ch}_{P}\left(\alpha_{\gamma}\right)$; a similar statement holds in the odd case. This provides a simple conceptual framework for the transgression result of Connes-Moscovici [5] in the case of bounded Fredholm modules.

\section{References}

[1] N. Bourbaki, Topological Vector Spaces, Chapters 1-5, Springer Verlag, Berlin 1987.

[2] A. Connes, Noncommutative differential geometry, Inst. Hautes Etudes Sc. Publ. Math., 62 (1985), 257-360

[3] A. Connes, Entire cyclic cohomology of Banach algebras and Chern characters of $\theta$-summable Fredholm modules, $K$-theory 1 (1988), 519-548.

[4] A. Connes, Noncommutative Geometry, Academic Press, San Diego, 1994.

[5] A. Connes, H. Moscovici, Transgression and the Chern character of finitedimensional K-cycles, Comm. Math. Phys. 155 (1993), no. 1, 103-122.

[6] J. Cuntz, Bivariante $K$-Theorie für localkonvexe Algebren und der ChernConnes-Charakter, Doc. Math. 2 (1997), 139-182.

[7] J. Cuntz, G. Skandalis, B. Tsygan, Cyclic Homology in Noncommutative Geometry, Encyclopaedia of Mathematical Sciences, v. 121, Springer Verlag, 2004. 
[8] J. Cuntz, D. Quillen, On excision in periodic cyclic cohomology. C. R. Acad. Sci. Paris Sér. I Math. 317 (1993), no. 10, 917-922.

[9] J. Cuntz, D. Quillen, Cyclic homology and nonsingularity, Journal Amer. Math. Soc. 8 (1995) 373-442.

[10] J. Cuntz, D. Quillen, Exision in bivariant periodic cyclic cohomology, Invent. math. 127 (1997), 67-98.

[11] A. Grothendieck, Produits tensoriels topologiques et espaces nucléaires, Memoirs of the Amer. Math. Soc. 16 (1955).

[12] A. Ya. Helemskii, The homology of Banach and topological algebras, Kluwer Academic Publishers Group, Dordrecht, 1989. Translated from the Russian by Alan West.

[13] H. Hogbe-Nlend, Théorie des Bornologies et Application, LNM 213, Springer Verlag, Berlin, Heidelberg, New York 1971.

[14] C. Kassel, Caractère de Chern bivariant, K-Theory 3 (1989), 367-400.

[15] M. Khalkhali, Algebraic connections, universal bimodules and entire cyclic cohomology. Comm. Math. Phys. 161 (1994), no. 3, 433-446.

[16] V. Mathai, D. Stevenson, Entire cyclic homology of stable continuous trace algebras, math.KT/0412485.

[17] R. Meyer, Analytic cyclic cohomology, Ph.D. Thesis, Münster 1999, math.KT/9906205.

[18] R. Meyer, Excision in entire cyclic cohomology. J. Eur. Math. Soc. (JEMS) 3 (2001), no. 3, 269-286.

[19] D. Perrot, Retraction of the bivariant Chern character, K-Theory 31(2004) $233-287$.

This article is available at http://intlpress.com/HHA/v7/n3/a3/

Jacek Brodzki j.brodzki@soton.ac.uk

School of Mathematical Sciences,

University of Southampton,

Southampton SO17 1BJ,

U.K.

Roger Plymen plymen@manchester.ac.uk

School of Mathematics,

University of Manchester,

Manchester M13 9PL,

U.K. 